\title{
On stationary points and the complementarity problem
}

\section{Sribatsa Nanda and Sudarsan Nanda}

Let $S$ be a closed convex cone in $C^{n}, S^{*}$ the polar cone, $g$ a continuous map from $c^{n}$ into itself, and $e$ a fixed vector in $S^{*}$. In this paper we prove that there is a connected set $T$ in $S$ of stationary points of $\left(D_{p}(e), g\right)$ where $D_{p}(e)$ is the set of all $x$ in $S$ with $r e(e, x) \leq r$. This extends the results of Lemke and Eaves to the complex nonlinear case and arbitrary closed convex cones in $C^{n}$. We show that if $g$ is strictly monotone on $S$, then $T$ is both unique as well as arcwise connected. This partly solves the open problems raised by Eaves in this more general setting. We also show that if $x$ is a stationary point of $\left(D_{r}(e), g\right)$ and $\operatorname{re}(e, x)<r$ then $x$ is a stationary point of $(S, g)$.

\section{Introduction}

Let $C^{n}\left(R^{n}\right)$ denote the $n$-dimensional complex (real) space with hermitian (euclidean) norm and the usual inner product and let $R_{+}^{n}$ be the nonnegative orthant of $R^{n}$. Let $S$ denote a closed convex cone in $C^{n}$. The polar of $S$, denoted by $S^{*}$, is defined by

$$
S^{*}=\left\{y \in C^{n}: \operatorname{re}(x, y) \geq 0 \text { for all } x \in S\right\} \text {. }
$$

Since $e \in S^{*}$ and $r \geq 0$, we write

Received 1 December 1978. 


$$
\begin{aligned}
& D_{r}(e)=\{x \in S: \operatorname{re}(e, x) \leq r\}, \\
& D_{r}^{0}(e)=\left\{x \in D_{r}(e): \operatorname{re}(e, x)<r\right\},
\end{aligned}
$$

and

$$
S_{p}(e)=\left\{x \in D_{r}(e): \operatorname{re}(e, x)=r\right\}
$$

Note that $D_{p}(e)$ is the disjoint union of $D_{r}^{0}(e)$ and $S_{p}(e)$. A mapping $g: C^{n} \rightarrow C^{n}$ is said to be monotone on $S$ if $\operatorname{re}(g(x)-g(y), x-y) \geq 0$ for each $(x, y) \in S \times S$ and strictly monotone if strict inequality holds whenever $x \neq y$.

Given a continuous function $g: c^{n}+c^{n}$, the nonlinear complementarity problem in $C^{n}$ consists of finding a $z$ such that

$$
z \in S, g(z) \in S^{*} \text {, and } \operatorname{re}(g(z), z)=0 \text {, }
$$

where $S$ is a closed convex cone in $c^{n}$. Given a convex set $k \subset c^{n}$ and a continuous map $g: c^{n} \rightarrow c^{n}$, a point $x \in K$ is said to be a stationary point of the pair $(K, g)$ if

$$
x \in \underset{y \in R}{\arg \min } \operatorname{re}(g(x), y)
$$

or equivalently, if

$$
x \in \underset{y \in K}{\arg \min }\|y-x+g(x)\|,
$$

where arg min means the set of all $y \in K$ which minimize the objective. Notice that the set in (1.3) is either empty or singleton, whereas in (1.2) it may contain many elements. Note that the existence of a solution to the complementarity problem (1.1) is equivalent to the existence of a stationary point of $(S, g)$.

Several authors including Bazaraa, Goode, and Nashed [1], Habetler and Price [4], Karamardian [6], Mond [8], and Parida and Sahoo [10] have discussed the solution of the complementarity problems in different contexts. Lemke [7] and Eaves [3] have discussed the existence of stationary points of $(S, g)$ in the real case by taking $S$ to be $R_{+}^{n}$. A basic theorem of Lemke [7] asserts that given an affine function 
$g: R_{+}^{n} \rightarrow R^{n}$ and a $d \in R_{+}^{n}$, there is a piecewise affine function $x: R_{+}^{1} \rightarrow R_{+}^{n}$ such that $x(t)$ is a stationary point of $\left(D_{t}^{n}, g\right)$ with $d \cdot x(t)=t$, where $D_{t}^{n}$ is the set of all $x$ in $R_{+}^{n}$ for which $d \cdot x \leq t$. The set $T=\{x(t)\}$ thus becomes connected (indeed, arcwise connected). While extending this result to the nonlinear case, Eaves [3] has shown that the arcwise connectedness property of $T$ is false in general and has asked the following two questions. What conditions give the arcwise connectedness property and what properties give a unique $T$ ?

In this paper we extend the results of Lemke [7] and Eaves [3] to the complex nonlinear case by taking $S$ to be any closed convex cone in $c^{n}$. We show that there is a connected set $T$ in $S$ such that each $x \in T$ is a stationary point of $\left(D_{p}(e), g\right)$ for some $r \geq 0$. We also show that if $g$ is strictly monotone on $S$, then $T$ is both unique as well as arcwise connected and this partly answers the questions raised by Eaves [3] in this more general setting. Finally we prove the existence of stationary points of $(S, g)$ under certain suitable conditions.

\section{Preliminary results}

We start by mentioning some useful results which will be needed in the proof of our main theorems. The following proposition is the complex version of a lemma of Hartman and Stampacchia [5]; we omit its proof since it involves the same arguments as in the real case. For an outline of the proof in the real case, see [3].

PROPOSITION 2.1. Let $g: c^{n}+c^{n}$ be a continuous map on a nonempty, compact, convex set $K \subset c^{n}$. Then $(K, g)$ has a stationary point.

The following lemma has been proved in [9].

LEMMA 2.2. Let $S$ be a closed convex cone in $c^{n}$ and let $e \in \operatorname{int}\left(S^{*}\right)$ be fixed. Then for any $r \geq 0$, the set $D_{r}(e)$ is compact and convex.

The following proposition is the complex version of a theorem of Browder (see [2]). 
PROPOSITION 2.3. Let $G: K \times[0, r] \rightarrow K$ be a continuous function, where $K$ is a nonempty, compact, convex set in $c^{n}$ and $[0, r]$ denotes the closed interval in $R^{1}$. Then there is a connected set $W$ in $K \times[0, r]$ intersecting both $K \times\{0\}$ and $K \times\{r\}$ such that $G(x, t)=x$ for alz $(x, t) \in W$.

Proof. Suppose that $K$ is a nonempty, compact, convex set in $C^{n}$. Let $h: C^{n} \rightarrow R^{2 n}$ be the linear homeomorphism of $C^{n}$ onto $R^{2 n}$. Then $K^{\prime}=h(K)$ is a nonempty, compact, convex set in $R^{2 n}$. Since $G: K \times[0, r] \rightarrow K$ is continuous, there is a continuous map $G^{\prime}: K^{\prime} \times[0, r] \rightarrow K^{\prime}$ defined by

$$
G^{\prime}(x, t)=h G\left(h^{-1}(z), t\right)
$$

for $(z, t) \in K^{\prime} \times[0, r]$. Therefore, by Browder's Theorem, there is a connected set $W^{\prime} \subset K^{\prime} \times[0, r]$ such that $W^{\prime} \cap\left(K^{\prime} \times\{0\}\right) \neq \varnothing$, $W^{\prime} \cap\left(K^{\prime} \times\{r\}\right) \neq \varnothing$, and $G^{\prime}(z, t)=z$ for all $(z, t) \in W^{\prime}$. Define a continuous map $\theta: K \times[0, r] \rightarrow K^{\prime} \times[0, r]$ by the rule

$$
\theta(x, t)=(h x, t) \text {, }
$$

and take $W=\theta^{-1}\left(W^{\prime}\right)$. Clearly $W$ is connected, $W \subset K \times[c, r]$, and $G(x, t)=x$ for all $(x, t) \in W$. Since $W^{\prime} \cap\left(K^{\prime} \times\{0\}\right) \neq \varnothing$, there is a $w^{\prime}=\left(z^{\prime}, 0\right) \in W^{\prime} \cap\left(K^{\prime} \times\{0\}\right)$, and then

$$
\theta^{-1}\left(w^{\prime}\right)=\left(h^{-1}\left(z^{\prime}\right), 0\right) \in \forall n(K \times\{0\}) \text {. }
$$

Thus $W \cap(K \times\{0\}) \neq \emptyset$ and similarly it can be shown that $W \cap(K \times\{r\}) \neq \varnothing$. This completes the proof.

LEMMA 2.4. Let $g: c^{n} \rightarrow c^{n}$ be a continuous map on a closed convex cone $S$ and let $e \in S^{*}$. If $x$ is a stationary point of $\left(D_{r}(e), g\right)$ for some $r \geq 0$, then $\operatorname{re}(g(x), x) \leq 0$. In this case if $x \in D_{r}^{0}(e)$ then $\operatorname{re}(g(x), x)=0$.

Proof. Suppose that $x$ is a stationary point of $\left(D_{p}(e), g\right)$. Then

$$
\operatorname{re}(g(x), x) \leq \operatorname{re}(g(x), z) \text { for all } z \in D_{r}(e) \text {. }
$$

In particular, 


$$
\operatorname{re}(g(x), x) \leq \frac{2}{2} \operatorname{re}(g(x), x) .
$$

Therefore $\operatorname{re}(g(x), x) \leq 0$. Now, if $x \in D_{p}^{0}(e)$, then there is some $\lambda>1$ such that $\lambda x \in S_{p}(e)$. Then we have

$$
\operatorname{re}(g(x), x) \leq \lambda \operatorname{re}(g(x), x) \text {. }
$$

This is impossible unless $\operatorname{re}(g(x), x)=0$.

LEMMA 2.5. Let $g: c^{n} \rightarrow c^{n}$ be a continuous map on a closed convex cone $S$ and $e \in S^{*}$. If $x$ is a stationary point of $\left(D_{r}(e), g\right)$ and $x \in D_{r}^{0}(e)$, then $x$ is a stationary point of $(S, g)$.

Proof. Let $x \in D_{p}^{0}(e)$. Then, from Lemma 2.4, it follows that $\operatorname{re}(g(x), x)=0$. Let $w \in S$; then $w=\lambda z$ for some $\lambda \geq 0$ and $z \in D_{p}(e)$. Since $x$ is a stationary point of $\left(D_{r}(e), g\right)$ we have

$$
\operatorname{re}(g(x), x) \leq \operatorname{re}(g(x), z) \text { for all } z \in D_{p}(e) \text {. }
$$

Since $\operatorname{re}(g(x), x)=0$ it follows from (2.1) that

$$
\operatorname{re}(g(x), x) \leq \operatorname{re}(g(x), w) .
$$

Thus $x$ is a stationary point of $(S, g)$.

\section{The main theorems}

We are now ready to prove our main theorem.

THEOREM 3.1. Let $g: c^{n} \rightarrow c^{n}$ be a continuous map on a closed convex cone $S \subset C^{n}$ and let $e \in \operatorname{int}\left(S^{*}\right)$. Then there is a closed connected set $T$ in $S$ such that

(i) for every $x \in T$ there is an $r \geq 0$ such that $x \in S_{r}(e)$ and $x$ is a stationary point of $\left(D_{r}(e), g\right)$, and

(ii) for each $r \geq 0$ there is an $x \in T$ such that $x$ is a stationary point of $\left(D_{p}(e), g\right)$.

Proof. It follows from Proposition 2.1 and Lerma 2.2 that $\left(D_{p}(e), g\right)$ 
has a stationary point for each $r \geq 0$. Let $T_{r}$ be the set of all stationary points of $\left(D_{p}(e), g\right)$ and let $T$ be the connected component of $\underset{r \geq 0}{\mathrm{U}} T_{r}$ which contains $0 \in C^{n}$. Clearly $T$ is closed and is the maximal connected set containing 0 and satisfying $(i)$. We now show that $T$ satisfies $(i i)$. Let $G: D_{r}(e) \times[0, r] \rightarrow D_{r}(e)$ be defined by

$$
G(x, t)=\underset{y \in D_{t}(e)}{\arg \min \|y-x+g(x)\|}
$$

Clearly $G$ is continuous. By Proposition 2.3 it follows that there is a connected set $W$ in $D_{r}(e) \times[0, r]$ which contains $(0,0)$ and $(x, r)$ for some $x \in T_{r}$. But $T$ contains the connected set $\{y:(y, t) \in W\}$, and hence $x \in T$.

THEOREM 3.2. Let $g: C^{n} \rightarrow C^{n}$ be continuous and strictly monotone on a closed convex cone $S$ and let $e \in \operatorname{int}\left(S^{*}\right)$. Then the set $T$ of Theorem 3.1 is unique; it is also arcwise connected.

Proof. Assume that $x_{1}$ and $x_{2}$ are two stationary points of $\left(D_{r}(e), g\right)$ for some $r>0$. Then we have

$$
\operatorname{re}\left(g\left(x_{1}\right), x_{1}-x_{2}\right) \leq 0
$$

and

$$
\operatorname{re}\left(g\left(x_{2}\right), x_{2}-x_{1}\right) \leq 0
$$

By adding (3.1) and (3.2) we get

$$
\operatorname{re}\left(g\left(x_{1}\right)-g\left(x_{2}\right), x_{1}-x_{2}\right) \leq 0 \text {. }
$$

Since $g$ is strictly monotone, this is impossible unless $x_{1}=x_{2}$. This proves the uniqueness of $T$.

To show that $T$ is arcwise connected, it is enough to show that the correspondence $r \mapsto x_{r}$ is a continuous map, where $x_{r}$ is the unique stationary point of $D_{p}(e)$. It will therefore suffice to show that if $r_{n} \rightarrow r \quad\left(\right.$ in $\left.R_{+}^{l}\right)$, then $x_{r_{n}} \rightarrow x_{r}$ in $S$. Let $r_{n} \rightarrow r^{-}$and consider the 
sequence $\left\{x_{r_{n}}\right\}$ in $S$. Notice first of all that $x_{r_{n}}$ is a stationary point of $D_{r_{n}}(e)$; moreover, if $r_{n}<r_{n+1}$, then $D_{r_{n}}(e) \subset D_{r_{n+1}}(e)$. Thus it follows that $\left\{x_{r_{n}}\right\} \subset D_{p}(e)$. Since $D_{p}(e)$ is compact, there is a subsequence $\left\{x_{p_{m}}\right\}$ which converges to $x$ in $D_{r}(e)$. Suppose that $x \in D_{p}^{0}(e)$; then we can find an $\varepsilon>0$ such that $x \in D_{r-\varepsilon}(e)$. Since $r_{n} \rightarrow r$, it follows that all except a finite number of $x_{r_{m}}$ 's will lie outside $D_{r-\varepsilon}(e)$; thus $x$ cannot be a point of accumulation. This contradiction shows that $x \in S_{r}(e)$. Since $x_{p}$ is the unique stationary point of $D_{p}(e)$ we have

$$
\operatorname{re}\left(g\left(x_{p}\right), x_{p}-y\right) \leq 0 \text { for all } y \in D_{r}(e) .
$$

We shall now show that

$$
\operatorname{re}(g(x), x-y) \leq 0 \text { for all } y \in D_{p}(e) .
$$

If $y \in D_{p}^{0}(e)$ then we have

$$
\operatorname{re}\left(g\left(x_{r_{m}}\right), x_{r_{m}}-y\right) \leq 0
$$

Since $g$ is continuous, taking the limits as $r_{m} \rightarrow r$ we get

$$
\operatorname{re}(g(x), x-y) \leq 0 \text {. }
$$

If, however, $y \in S_{r}(e)$, then given $x_{r_{m}}$ we can choose $\lambda_{m} \in[0,1]$ such that $\lambda_{m} y \in D_{r_{m}}(e) ;$ thus

$$
\operatorname{re}\left(g(x), x-\lambda_{m} y\right) \leq 0
$$

Notice that as $r_{m} \rightarrow r, \lambda_{m} \rightarrow 1$, and thus taking the limit as $\lambda_{m} \rightarrow 1$, we get

$$
\operatorname{re}(g(x), x-y) \leq 0
$$

Therefore (3.4) holds, and combining (3.3) and (3.4) we have that $x=x_{r}$. 
This shows that all convergent subsequences of the sequence $\left\{x_{r_{n}}\right\}$ will have the same limit $x_{r}$, and therefore the sequence $\left\{x_{r_{n}}\right\}$ converges to $x_{r}$.

Now if $r_{n} \rightarrow r^{+}$, we can choose an $s>0$ such that $s>r_{n}$ for all $n$. Now since $D_{s}(e)$ is compact we can go through the same argument as in the case above to show that $x_{r_{n}} \rightarrow x_{r}$. This completes the proof that $r \mapsto x_{r}$ is continuous.

In order to show the existence of a stationary point of $(S, g)$, we introduce the following definition.

DEFINITION. Let $D$ be a subset of $S$. We say that a bounded set $U \subset S \cap D^{c}$ separates $D$ from $\infty$ if each unbounded closed connected set in $S$ that meets $D$ also meets $U$.

THEOREM 3.3. Let $g: c^{n} \rightarrow c^{n}$ be a continuous map on $S$ and let $e \in \operatorname{int}\left(S^{*}\right)$. Suppose that $U$ separates $D_{r}(e)$ from $\infty$ and that for each $x \in U$ there is a $w \in D_{r}(e)$ for which $\operatorname{re}(g(x), w) \leq \operatorname{re}(g(x), x)$. Then $(S, g)$ has a stationary point.

Proof. Let $T$ be as in Theorem 3.1. If $T \cap U=\varnothing$, then $T$ is bounded and therefore the result holds. Assume that $x \in T \cap U$. Since $x \in T$, by Theorem 3.1, there is a $k>0$ such that $x \in S_{k}(e)$ and $x$ is a stationary point of $D_{k}(e)$. If $k<r$, then clearly $x \in D_{r}^{0}(e)$ and by Lemma 2.4, $r e(g(x), x)=0$. Now assume that $r \leq k$. Then we have

$$
\operatorname{re}(g(x), x) \leq \operatorname{re}(g(x), z)
$$

for all $z \in D_{k}(e)$. Since $x \in U$ by the hypothesis, there is a

$w \in D_{p}(e)$ for which

$$
\operatorname{re}(g(x), w) \leq \operatorname{re}(g(x), x) .
$$

From (3.5) and (3.6) it follows that

$$
\operatorname{re}(g(x), w) \leq \operatorname{re}(g(x), z)
$$


for all $z \in D_{k}(e)$. Since $r \leq k, D_{r}(e) \subset D_{k}(e)$, and therefore $w \in D_{k}(e)$. If we take $z=x / 2+w$ in (3.7), we get

$$
\operatorname{re}(g(x), x) \geq 0 \text {. }
$$

Now, from (3.8) and Lemma 2.4, it follows that $\operatorname{re}(g(x), x)=0$. The required result then follows from Lemma 2.5 .

\section{References}

[1] M.S. Bazaraa, J.J. Goode and M.Z. Nashed, "A nonlinear complementarity problem in mathematical programing in Banach space", Proc. Amer. Math. Soc. 35 (1972), 165-170.

[2] Felix E. Browder, "On continuity of fixed points under deformations of continuous mappings", Summa Brasil. Math. 4 (1960), 183-191.

[3] B.C. Eaves, "On the basic theorem of complementarity", Math. Progrcoming 1 (1971), 68-75.

[4] G.J. Habetler and A.L. Price, "Existence theory for generalized nonlinear complementarity problems", J. Optimization Theory App Z. 7 (1971), 223-239.

[5] Philip Hartman and Guido Stampacchia, "On some non-linear elliptic differential-functional equations", Acta Math. 115 (1966), $271-310$.

[6] S. Karamardian, "Generalized complementarity problem", $J$. Optimization Theory Appl. 8 (1971), 161-168.

[7] C.E. Lemke, "Bimatrix equilibrium points and mathematical programming", Management Sci. Ser. A 11 (1965), 681-689.

[8] Bertram Mond, "On the complex complementarity problem", BuZz. Austral. Math. Soc. 9 (1973), 249-257.

[9] Sribatsa Nanda and Sudarsan Nanda, "A complex nonlinear complementarity problem", Bull. Austral. Math. Soc. 19 (1978), 437-444 (1979). 
[10] J. Parida and B. Sahoo, "On the complex nonlinear complementary problem", Bull. Austral. Math. Soc. 14 (1976), 129-136.

Department of Mathematics,

Regional Engineering College,

Rourkela,

Orissa,

India. 